MAROUELLI, W.A.; SILVA, W.L.C.; SILVA, H.R.; VILELA, N.J. Eficiência econômica do manejo racional da irrigação em tomateiro para processamento industrial. Horticultura Brasileira, Brasília, v. 18, n. 3, p. 238-243, novembro 2.000.

\title{
Eficiência econômica do manejo racional da irrigação em tomateiro para processamento industrial.
}

\author{
Waldir Aparecido Marouelli; Washington L.C. Silva; Henoque R. Silva; Nirlene J. Vilela
}

Embrapa Hortaliças, C. Postal 218, 70.359-970 Brasília - DF. e.mail: waldir@cnph.embrapa.br

\section{RESUMO}

A água é um dos principais fatores que afetam a produção e a qualidade de frutos do tomateiro. Embora existam inúmeras tecnologias disponíveis para o manejo racional da irrigação, a grande maioria dos produtores ainda irriga de forma inadequada. Um método simples, prático e criterioso para manejo é o do balanço de água no solo com base no tanque classe A. Objetivou-se neste estudo avaliar a vantagem econômica gerada pelo uso do tanque classe A para o manejo da irrigação do tomateiro na região do Brasil Central. Simulações foram realizadas utilizando-se sistemas por aspersão convencional portátil e pivô central para áreas irrigadas até 100 ha. A introdução da tecnologia gerou incrementos da receita líquida de $\mathrm{R} \$ 70,14$ a 263,49/ha para pivô central e de R \$ 109,98 a 303,33/ha para aspersão convencional portátil para áreas de 3 a 100 ha, respectivamente. O tempo para o retorno do investimento com a tecnologia foi inferior a um ano para áreas acima de 10 ha. Para áreas menores que 3 ha a utilização da tecnologia mostrou-se economicamente inviável.

Palavras-chave: Lycopersicon esculentum, manejo de água, análise econômica.

\section{ABSTRACT}

Economical efficiency of sound irrigation scheduling technique for processing tomatoes.

Water is one of the major factors affecting tomato yield and quality. In spite of several irrigation scheduling methods available, most growers still irrigate in an empirical way. A simple, practical and sound technique is the soil water balance with a class A pan. The objective of this study was to evaluate the economical advantages from the use of class A pan to schedule irrigation for tomato crop in Central Brazil. Simulations were performed considering hand-move and center pivot sprinkle systems for areas up to 100 ha. Net income resulting from the use of the technology increased from R $\$ 70.14$ to 263.49 /ha for central pivot and from R\$ 109.98 to 303.33 /ha for hand-move system for areas from 3 to 100 ha, respectively. The payback of the technology investment was less than one year for irrigated areas over 10 ha. For areas under 3 ha the technology was found not economically viable.

Keywords: Lycopersicon esculentum, irrigation scheduling, economical analysis.

(Aceito para publicação em 06 de setembro de 2.000)

$\mathrm{T}$ omate para processamento industrial é a hortaliça mais importante da indústria agroalimentar brasileira, com um crescimento de mercado estimado em $10 \%$ ao ano. A área total cultivada em 1998 foi de aproximadamente 22.280 ha, sendo cerca de $50 \%$ localizada na região de cerrados do Brasil Central, principalmente Goiás e Minas Gerais (ABIA, 1999*). A cultura é qua- se que totalmente irrigada, sendo a aspersão o principal método utilizado. No Nordeste e em São Paulo os sistemas mais comuns são os convencionais portáteis enquanto no Brasil Central o pivô 
central é o sistema mais utilizado (Barbosa, 1997).

A água é um dos fatores mais importantes que afetam a produtividade $\mathrm{e}$ a qualidade industrial de frutos de tomate. Assim, o controle adequado da irrigação constitui fator preponderante para o êxito da cultura, maximizando a produtividade, a eficiência no uso de água e de nutrientes e minimizando os custos de irrigação, tornando a atividade mais lucrativa (Marouelli et al., 1991; Battilani, et al., 1999). Embora existam inúmeras tecnologias disponíveis para manejo racional da irrigação do tomateiro industrial (Choudhury et al., 1980; Marouelli et al., 1991; Marouelli et al., 1993), a grande maioria dos produtores ainda irriga de forma inadequada (Silva \& Marouelli, 1996), ou seja, a decisão de quando e quanto irrigar não é baseada em parâmetros quantitativos relacionados à dinâmica de água no sistema solo-planta-atmosfera, mas apenas em observações visuais da cultura e da camada superficial do solo. O baixo índice de adoção das tecnologias deve-se principalmente ao fato dos agricultores acreditarem que estas são caras, complicadas, trabalhosas e, sobretudo, sem resultados que proporcionem ganhos financeiros compensadores.

Dentre os problemas associados ao manejo inadequado da irrigação destacam-se menores produtividades, frutos de qualidade inferior, maior incidência de doenças, maior uso de energia e danos ao meio ambiente (Marouelli et al., 1991; Silva et al., 1999). Particularmente na região do Brasil Central, a utilização de pivô central vem sendo prejudicada pelo manejo inadequado da água, pela falta de esquema eficiente de rotação de culturas e severa ocorrência de Sclerotinia sclerotiorum, fungo causador da doença podridão-de-esclerotínea. Além disso, a ocorrência das bactérias Xanthomonas campestris pv. vesicatoria e Pseudomonas syringae pv. tomato, favorecida pela irrigação por aspersão, vem causando reduções significativas de produtividade e qualidade de frutos (Silva et al., 1997).

Vários são os métodos existentes para manejo racional da irrigação nas diferentes culturas, como o da tensão e o do balanço de água no solo. Variações desses métodos existem dependendo de cada condição e instrumentos utilizados (Marouelli et al., 1996). Dentre estes, o método do balanço de água, com base no tanque classe A, é simples, prático, criterioso e apresenta melhor potencial para manejo da irrigação em tomateiro industrial (Roque et al., 1986; Silva \& Marouelli, 1996). O uso de tensiômetros para controle da irrigação em tomateiro não é adequado por serem as tensões críticas recomendadas para manejo de água na cultura maiores que aquelas possíveis de serem medidas pelo instrumento (Choudhury et al., 1980; Marouelli et al., 1991). Além de mais simples, métodos baseados no balanço de água são de custo mais baixo que aqueles baseados no status de água no solo (Tan, 1988).

Este estudo teve como objetivo demonstrar a vantagem econômica gerada pela introdução da tecnologia de manejo racional da irrigação, baseada no uso do tanque classe $\mathrm{A}$, em tomateiro para processamento industrial na região de cerrados do Brasil Central.

\section{MATERIAL E MÉTODOS}

Para a análise das vantagens econômicas resultantes da modificação no sistema de produção de tomate para processamento industrial, causada pela introdução da tecnologia de manejo racional da irrigação, foi desenvolvido um modelo de simulação (Figura 1). Com base em premissas teóricas consistentes, as simulações foram realizadas utilizando-se sistemas de irrigação por aspersão convencional portátil e pivô central para áreas irrigadas variando de $1 \mathrm{a}$ 100 ha e de 3 a 100 ha, respectivamente. Os cálculos foram realizados em termos de período produtivo (uma safra por ano), ou seja, não foi considerada a irrigação de nenhuma outra cultura na entressafra do tomate.

O manejo tradicional da irrigação utilizado pelos tomaticultores da região de cerrados do Brasil Central, chamado aqui de "manejo inadequado" (MI), é em geral realizado de forma empírica sem nenhum embasamento teórico. Em termos médios, as irrigações são feitas a cada 4 dias e a quantidade líquida de água aplicada por irrigação é de $16 \mathrm{~mm}$ até o início de florescimento e de $24 \mathrm{~mm}$ até cerca de 5 dias antes da colheita, quando as irrigações são suspensas (Silva $\&$ Marouelli, 1996).

O método de manejo da irrigação recomendado pela Embrapa Hortaliças para tomateiro industrial, chamado aqui de "manejo racional" (MR), é o do balanço de água no solo. A quantidade de água aplicada por irrigação é calculada com base na evaporação do tanque classe A (Marouelli et al., 1996), utilizando-se coeficientes de cultura apresentados por Marouelli \& Silva (1993). As irrigações são realizadas a cada 5-7 dias (Silva \& Marouelli, 1996) e paralisadas quando $50 \%$ dos frutos apresentaremse totalmente maduros (Marouelli \& Silva, 1993).

O custo total anual incluiu os custos fixos e variáveis do sistema de irrigação e dos instrumentos para manejo da irrigação e o custo de produção da cultura. Ao custo de produção foram incluídos todos os custos com mecanização, como tratores e implementos agrícolas, utilizados no cultivo do tomateiro. A receita total foi igual ao valor de venda da produção comercial. O custo fixo total anual foi computado pela soma da depreciação $\left(\mathrm{D}_{\mathrm{ma}}\right)$ mais o custo de oportunidade do capital $\left(\mathrm{J}_{\mathrm{ma}}\right)$ sobre o valor do investimento.

$$
\begin{aligned}
& \mathrm{D}_{\mathrm{ma}}=\frac{\text { Vlr. compra - Vlr. venda }}{\text { vida útil (anos) }} \\
& \mathrm{J}_{\mathrm{ma}}=\frac{\text { Tx. juros Vlr.compra }+ \text { Vlr.venda }}{100} \mathrm{x} \frac{2}{2}
\end{aligned}
$$

Dentre os custos variáveis têm-se os operacionais, de manutenção e adicionais. Os custos operacionais referiramse às despesas com energia e mão-deobra para operação do sistema de irrigação e para manejo da irrigação (leitura de instrumentos, manutenção e cál-

\footnotetext{
* Associação Brasileira das Indústrias Alimentícias. São Paulo, SP.
} 


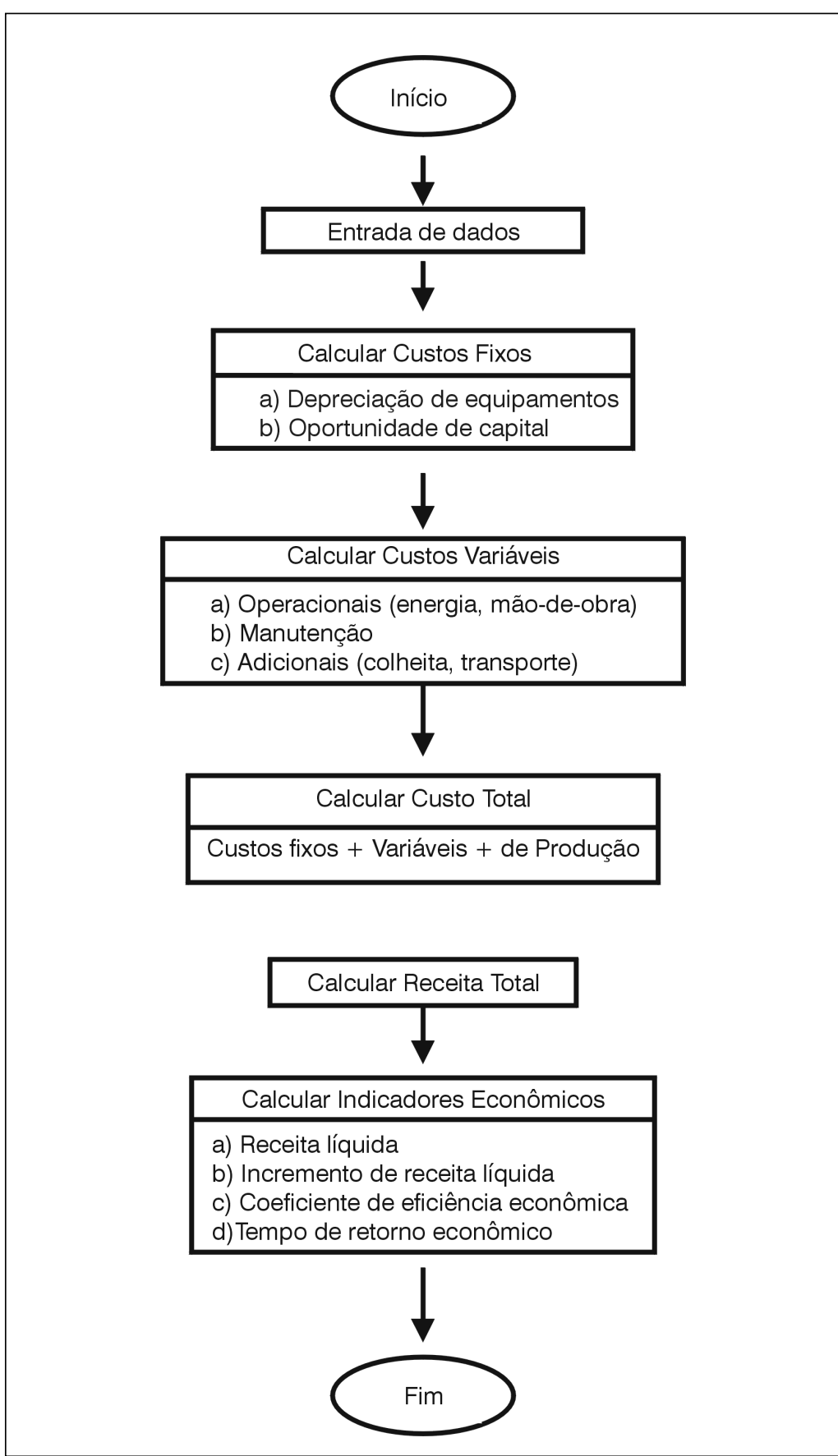

Figura 1. Fluxograma do modelo para análise da eficiência econômica referente a introdução da tecnologia para manejo racional da irrigação. Brasília, Embrapa Hortaliças, 1999.

culos). O custo de manutenção do sistema de irrigação, que incluiu os gastos com peças de reposição, mão-de-obra e lubrificantes, foi calculado como uma fração do valor de compra do equipamento (Tabela 1). Custos adicionais constaram das despesas com colheita e transporte decorrentes do aumento de produção oriundos da introdução do método MR para manejo da irrigação.

Adotou-se a taxa de juros equivalente $6 \%$ ao ano, conforme praticada atualmente no mercado de capitais e adotada pela Fundação Getúlio Vargas para análise de projetos. O custo de mão-deobra para operação dos sistemas de irrigação foi de R \$ 2,50/h-homem, de R\$ $5,00 /$ h-homem para manejo, de $\mathrm{R} \$$ $15,00 /$ t para colheita manual e de R\$ 12,00/t para transporte (distância de 100 $\mathrm{km})$. A tarifa de energia elétrica, considerando demanda de potência, foi de R\$ $0,055 / \mathrm{kWh}$ para pivô central $(50 \%$ da irrigação no horário noturno; $80 \%$ de desconto na tarifa) e de $\mathrm{R} \$ 0,095 / \mathrm{kWh}$ para o convencional portátil $(100 \%$ da irrigação no horário diurno). O preço do produto pago ao agricultor foi de $\mathrm{R} \$$ $70,00 / \mathrm{t}$ e o ciclo de irrigação de 95 dias.

O custo dos instrumentos necessários para utilização do método do balanço de água no solo totalizou $\mathrm{R} \$ 2.400,00$ (pluviômetro "Ville de Paris", tanque classe A em aço inoxidável, micrômetro de gancho, poço tranquilizador e estrado de madeira). A vida útil dos instrumentos foi estimada em 20 anos.

O custo de aquisição do sistema de irrigação convencional portátil com aspersores tipo canhão (raio de alcance $35 \mathrm{~m}$ ) foi assumido como independente do tamanho da área irrigada, igual a R\$ $1.000,00 /$ ha. Para pivô central, os custos considerados foram de $\mathrm{R} \$ 4.500,00$; $3.000,00 ; 2.100,00 ; 2.000,00 ; 1.900,00$; $1.800,00 ; 1.700,00 ; 1.600,00 ; 1.550,00$; $1.500,00$ e 1.450,00/ha para áreas de 3 , $10,20,30,40,50,60,70,80,90$ e 100 ha, respectivamente.

Dados adicionais necessários para a análise econômica são apresentados na Tabela 1. Os dados de custos utilizados na análise foram obtidos através de consultas às indústrias de processamento, empresas de equipamentos de irrigação e produtores. Informações sobre vida útil de sistemas de irrigação, uso de mão-de-obra, uso de energia e custos de manutenção foram obtidos de Marouelli $\&$ Silva (1998). Assumiu-se que a produtividade e os custos de produção por unidade de área foram independentes do tamanho da área cultivada. O valor de venda ou de sucata dos sistemas e instrumentos para controle da irrigação foi considerado desprezível. Todos os custos e índices financeiros utilizados na análise foram referentes a dezembro de 1998.

Valores de produtividade de frutos para os sistemas por aspersão convencional portátil e pivô central, para as 
condições de manejo inadequado e racional, foram determinados com base em valores médios observados em áreas de produção comercial e experimentos conduzidos na Embrapa Hortaliças (Marouelli et al., 1991; Silva \& Marouelli, 1996). Muito embora a adoção do manejo MR possa reduzir o uso de pesticidas, os gastos com tais produtos foram considerados iguais para as condições com manejo MR e MI.

Como indicadores econômicos foram calculados a receita líquida para as condições com manejo MR e MI, dada pela diferença entre a receita e os custos totais; o incremento de receita líquida resultante da introdução da tecnologia, dado pela variação entre as receitas líquidas para as condições com manejo MR e MI; o coeficiente de eficiência econômica da tecnologia, dado pela relação entre o valor total do incremento de produção proporcionado pelo uso da tecnologia e o custo total anual da tecnologia (fixo e variável); e o tempo para retorno econômico do investimento ("payback") da tecnologia, dado pela relação entre o valor de aquisição dos instrumentos para manejo da irrigação e a receita anual líquida extra propiciada pela introdução da tecnologia.

\section{RESULTADOS E DISCUSSÃO}

Os resultados da análise econômica referentes à introdução do manejo MR para os dois sistemas de irrigação, em função do tamanho da área irrigada, são apresentados nas Figuras 2 a 4.

A receita líquida aumentou com a área irrigada, principalmente para áreas inferiores a 20 ha, tanto para o método MR quanto para o MI, com exceção para o sistema de irrigação convencional com método de manejo MI (Figuras 2 e 3). Para este caso, a receita líquida foi independente da área, o que se deve à hipótese assumida de que a produtividade, o custo de produção e o preço por hectare da aspersão convencional são independentes da área irrigada. Todos os sistemas de irrigação e métodos de manejo avaliados apresentaram receita líquida positiva (entre $\mathrm{R} \$ 409,82$ a $1.426,10 / \mathrm{ha})$, para as áreas consideradas, indicando serem economicamente viáveis. Verifica-se todavia, que a receita

Tabela 1. Índices adicionais utilizados na análise econômica. Brasília, Embrapa Hortaliças, 1999.

\begin{tabular}{|c|c|c|c|c|}
\hline \multirow[t]{2}{*}{ Componentes/fatores } & \multicolumn{2}{|c|}{ Manejo racional } & \multicolumn{2}{|c|}{$\begin{array}{c}\text { Manejo } \\
\text { inadequado }\end{array}$} \\
\hline & Pivô & Portátil & Pivô & Portátil \\
\hline Vida útil (anos) & 15 & 12 & 15 & 12 \\
\hline Uso de energia (kWh/m3) & 0,35 & 0,40 & 0,35 & 0,40 \\
\hline Eficiência de irrigação (\%) & 80 & 65 & 80 & 65 \\
\hline $\begin{array}{l}\text { Lâmina líquida de água } \\
\text { (mm/ciclo) }\end{array}$ & 400 & 400 & 510 & 510 \\
\hline Mão-de-obra (h/ha/irrigação) & 0,2 & 2,0 & 0,2 & 2,0 \\
\hline $\begin{array}{l}\text { Mão-de-obra para manejo } \\
\text { (h/dia) }\end{array}$ & 1,0 & 1,0 & 0,0 & 0,0 \\
\hline $\begin{array}{l}\text { Número de irrigações por } \\
\text { ciclo }\end{array}$ & 24 & 24 & 34 & 34 \\
\hline Custos de manutenção (\%) & 5,0 & 2,0 & 5,0 & 2,0 \\
\hline Custo de produção(R\$ /ha $)^{\mathrm{b}}$ & $3.035,00$ & $2.900,00$ & $3.035,00$ & $2.900,00$ \\
\hline Produtividade (t/ha) & 75 & 69 & 65 & 60 \\
\hline
\end{tabular}

a Percentagem do investimento inicial.

b Não inclui custos fixos e variáveis da irrigação e instrumentos para manejo, bem como custos adicionais resultantes do uso de tecnologia para manejo. O custo de $\mathrm{R} \$ 2.900,00$ inclui custos adicionais para colheita e transporte de $60 \mathrm{t} / \mathrm{ha}$, enquanto $\mathrm{R} \$ 3.035,00$ inclui custos de 65 t/ha.

Fonte: Adaptado de Silva \& Marouelli (1996) e Marouelli \& Silva (1998).

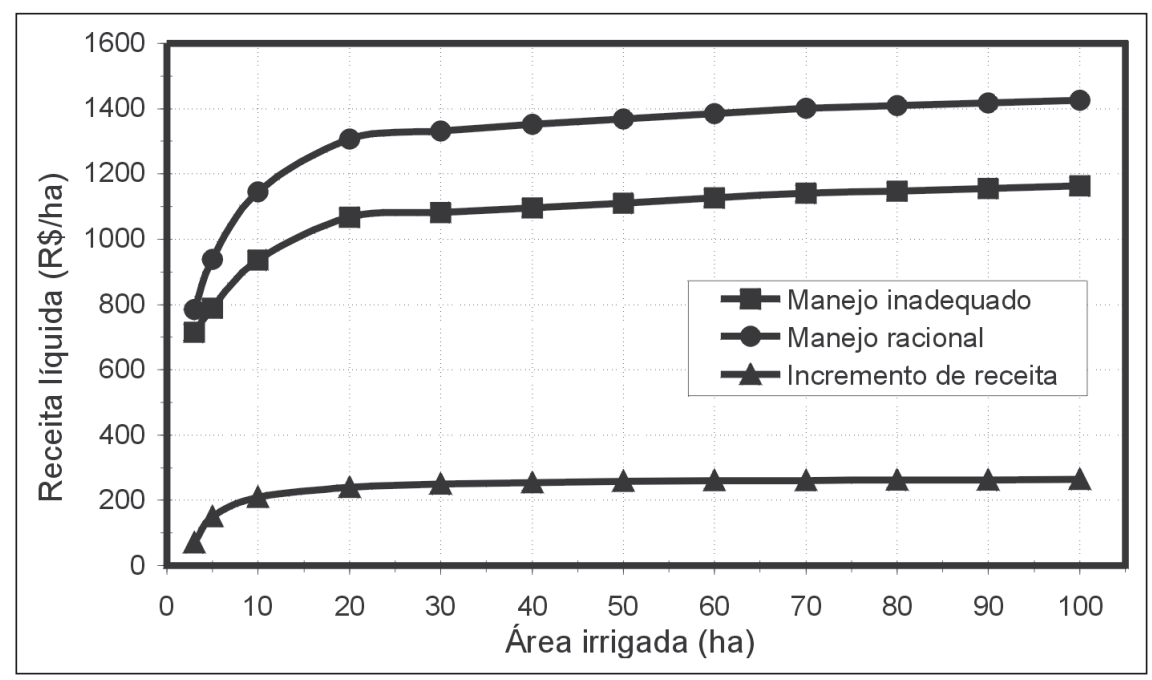

Figura 2. Receita e incremento de receita líquida na produção de tomate industrial irrigado por pivô central com manejo inadequado e racional em função da área irrigada. Brasília, Embrapa Hortaliças, 1999.

líquida para o sistema por aspersão convencional com manejo MR, para áreas inferiores a 3 ha, foi menor que a receita líquida para a condição de manejo inadequado (Figura 3).

$\mathrm{O}$ incremento de receita líquida, resultante do uso do método racional para manejo da irrigação, variou de R\$ 109,98 a $279,41 /$ ha para o sistema de irrigação convencional portátil e de R $\$$ 70,14 a 239,57/ha para pivô central, para áreas entre 3 e 20 ha, respectivamente. Acima de 20 ha o incremento foi praticamente constante, tendo atingido, para uma área de 100 ha, R\$ 303,33/ha para o sistema de irrigação convencional portátil e de R \$ 263,49/ ha para pivô central (Figuras 2 e 3 ). 


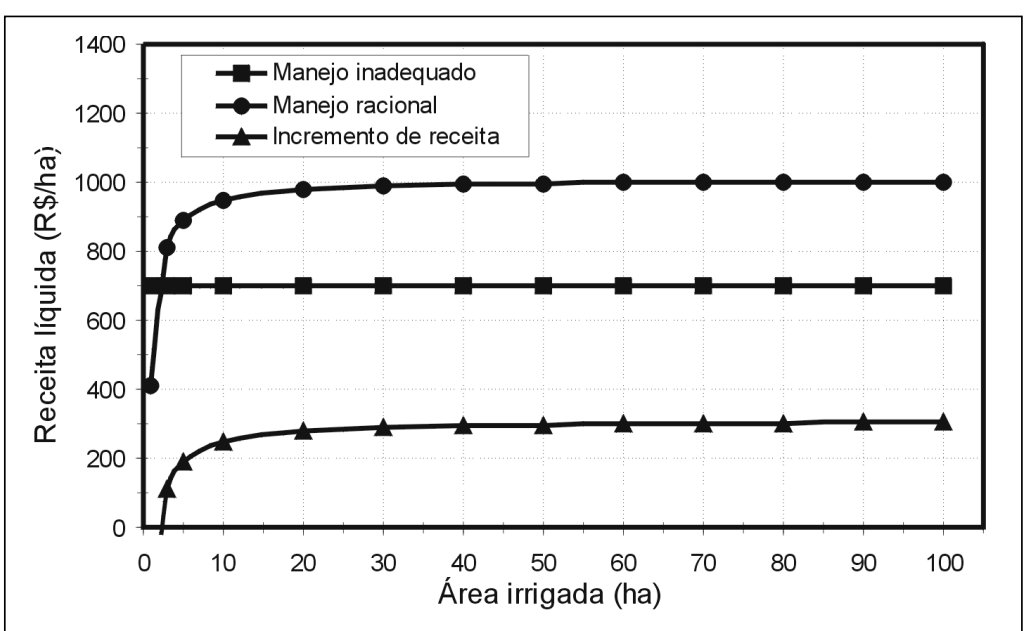

Figura 3. Receita e incremento de receita líquida na produção de tomate industrial irrigado por aspersão convencional portátil com manejo inadequado e racional em função da área irrigada. Brasília, Embrapa Hortaliças, 1999.

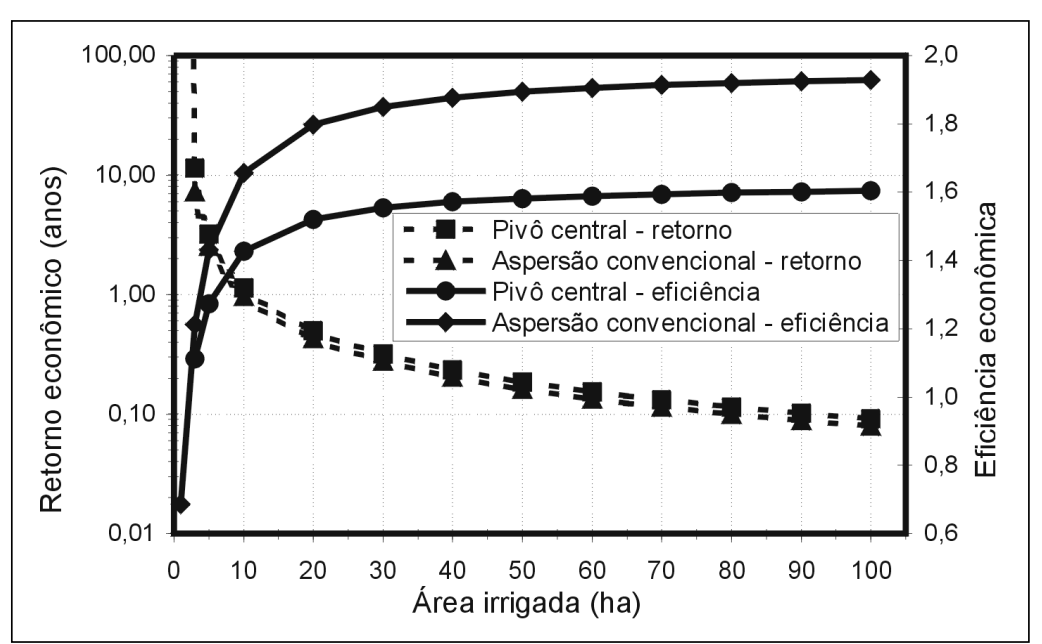

Figura 4. Coeficiente de eficiência econômica da tecnologia e tempo para retorno econômico do investimento para manejo racional da irrigação por aspersão convencional portátil e pivô central. Brasília, Embrapa Hortaliças, 1999.

O considerável aumento no incremento da receita líquida para áreas de 3 a 20 ha, seguido de uma quase estabilização (Figuras 2 e 3), deve-se aos custos para introdução da tecnologia para manejo MR (custos fixos e variáveis anuais iguais a R\$790,00) serem independentes da área irrigada.

O coeficiente de eficiência econômica da tecnologia, que representa a relação benefício/custo, aumentou de forma logarítmica com a área irrigada, atingindo um valor máximo, para $100 \mathrm{ha}$, de 1,93 unidades de benefícios para cada unidade de custo para o sistema conven- cional portátil e de 1,60 para o sistema pivô central (Figura 4). Para o sistema convencional com áreas inferiores a 3 ha, o incremento de receita líquida foi negativo e o coeficiente de eficiência econômica menor que a unidade (Figuras $3 \mathrm{e}$ 4), indicando que a soma dos custos fixos, referentes à aquisição dos instrumentos para manejo, e dos custos variáveis ção foram superiores ao incremento de receita resultante do uso da tecnologia. Assim, o uso da tecnologia para manejo MR mostrou ser economicamente inviável para áreas inferiores a 3 ha. com mão-de-obra para manejar a irriga-
O tempo para o retorno econômico do capital investido na introdução da tecnologia, indicando o número de anos (safras) necessários para recuperar o investimento para aquisição dos instrumentos para manejo MR, foi reduzido com o aumento da área irrigada (Figura 4). Independentemente do sistema de irrigação adotado, o tempo de retorno foi inferior a um ano para áreas irrigadas superiores a 10 ha. Para áreas menores que 10 ha o tempo de retorno aumentou rapidamente, tornando-se o investimento irrecuperável para áreas inferiores a 3 ha. Isto indica que o aumento de custos resultante da modificação no sistema de produção de tomate industrial, pela introdução do método para manejo baseado no uso do tanque Classe A, foi economicamente compensado pelo aumento de produção apenas para áreas irrigadas superiores a 3 ha

O presente estudo foi realizado para condições médias observadas no sistema de produção de tomate para processamento industrial na região de cerrados do Brasil Central. Portanto, resultados para situações específicas, como diferentes condições edafoclimáticas, nível de experiência do agricultor, incidência de pragas e doenças, podem diferir daqueles reportados neste estudo. Assim, por exemplo, é esperado que a vantagem econômica resultante da introdução da tecnologia para manejo MR seja relativamente maior para agricultores com pouca experiência no manejo da irrigação com a cultura do tomateiro e menor para aqueles com grande experiência.

\section{LITERATURA CITADA}

BARBOSA, V. The processing tomato growing system under tropical and subtropical conditions: the Brazilian experience. In: INTERNATIONAL CONFERENCE ON THE PROCESSING TOMATO, 1., 1996, Recife. Proceedings... Alexandria: ASHS/IPA, 1997. p. 94-97.

BATTILANI, A.; PIVA, A.; DADOMO, M. Evaluation of the decision support system for irrigation scheduling of processing tomato adopted in the Emilia-Romagna region. Acta Horticulturae, n. 487, p. 507-511, 1999.

CHOUDHURY, E.N.; MILLAR, A.A.; CHOUDHURY, M.M.; ABREU, T.A.S. Diferentes níveis de irrigação na produção e sistema radicular do tomate industrial. Pesquisa Agropecuária Brasileira, Brasília, v. 15, n. 4, p. $475-480,1980$. 
MAROUELLI, W.A.; SILVA, H.R.; OLIVEIRA, C.A.S. Produção de tomate industrial sob diferentes regimes de umidade no solo. Pesquisa Agropecuária Brasileira, Brasília, v. 26, n. 9, p. 1531-537, 1991.

MAROUELLI, W.A.; SILVA, W.L.C. Adequação da época de paralisação das irrigações em tomate industrial no Brasil Central. Horticultura Brasileira, Brasília, v. 11, n. 2, p. 218-221, 1993.

MAROUELLI, W.A.; SILVA, W.L.C. Seleção de sistemas de irrigação para hortaliças. Brasília: EMBRAPA-CNPH, 1998. 15 p. (Circular Técnica da Embrapa Hortaliças, 11).

MAROUELLI, W.A.; SILVA, W.L.C.; SILVA, H.R. Manejo da irrigação em hortaliças. Brasília: EMBRAPA-SPI/EMBRAPA-CNPH, 1996. $72 \mathrm{p}$.
ROQUE, R; LEON, M.; GONZALEZ, P; CORONA, Y.L. Utilizacion del evaporimetro Clase "A" para la determinacion del momento de riego en el tomate. Ciencia y Tecnica en la Agricultura: Riego y Drenaje, v. 9, n. 1, p. 45$55,1986$.

SILVA, W.L.C.; GIORDANO, L.B MAROUELLI, W.A.; FONTES, R.R.; GORNAT, B. Response of six processing tomatoes cultivars to subsurface drip fertigation. Acta Horticulturae, n. 487, p. 569573, 1999.

SILVA, W.L.C.; LOPES, C.A.; PEREIRA, W.; FONTES, R.R. Crop rotation systems for irrigated processing tomatoes in Central Brazil. In: INTERNATIONAL CONFERENCE ON THE PROCESSING TOMATO, 1; 1996, Recife. Proceedings... Recife: ASHS/IPA, 1997. p. 80 .
SILVA, W.L.C.; MAROUELLI, W.A. Evaluation of irrigation scheduling techniques for processing tomatoes in Brazil. In: INTERNATIONAL CONFERENCE ON EVAPOTRANSPIRATION AND IRRIGATION SCHEDULING, 1996, San Antonio. Proceedings... San Antonio: ASAE, 1996. p. 522-526.

TAN, C.S. Comparison of irrigation scheduling for processing tomatoes based on soil moisture measurement and climatological data. Acta Horticulturae, n. 228, p. 91-96, 1988. 